\title{
Going beyond Poisson processes: a new statistical framework in neuronal modeling and data analysis
}

\author{
Taşkın Deniz ${ }^{*}$, Stefan Rotter \\ From Twenty Second Annual Computational Neuroscience Meeting: CNS*2013 \\ Paris, France. 13-18 July 2013
}

Cortical spike trains in vivo often show a high irregularity, reflected by a coefficient of variation $(\mathrm{CV})$ close to one [1]. Such irregular single neuron spiking has consequently been associated with Poisson processes, and many models involving neuronal spike trains inherited this ideology. This viewpoint is further supported by the Palm-Khintchine theorem, which states that a superposition of a large number of renewal processes with very small intensity behaves like a Poisson process. It was demonstrated, however, that this theorem doesn't always apply to the superposition of neuronal spike trains [2-4]. Moreover, Poisson processes lack the temporal properties observed in population responses to input modulation either via a stimulus in vivo [5] or via electrical stimulation in vitro [6].

In this study, we report on new techniques to deal with non-Possonian aspects of stationary neuronal spike trains, as well as non-equilibrium population responses [7], based on Markov point processes (MPP), commonly known as continuous time Markov chains (CTMC). We compute the interspike interval (ISI) distribution by algebraically solving the first passage time problem for MPP neuron models, and compute the transient population responses with a similar technique. The same technique is used to compute exact cross-correlation functions for a shared input paradigm [8]. We advertise MPPs as a new powerful framework in neural network modeling and neural data analysis with many possible applications.

\section{Acknowledgements}

This work is supported by the German Federal Ministry of Education and Research (BMBF; grant 01GQ0420 to BCCN Freiburg and grant 01GQ0830 to BFNT Freiburg*Tubingen) and the German Research Foundation (DFG; grant EXC 1086 to the Cluster of Excellence BrainLinks-BrainTools).

Published: 8 July 2013

\section{References}

1. Shadlen MN, Newsome WT: The variable discharge of cortical neurons: implications for connectivity, computation and information coding. Journal of Neuroscience 1998, 18:3870-3896.

2. Lindner B: Superposition of many independent spike trains is generally not a Poisson process. Physical Review E 2006, 73:022901.

3. Câteau H, Reyes A: Relation between single neuron and population spiking statistics and effects on network activity. Physical Review Letters 2006, 96(5):058101.

4. Deger M, Helias M, Boucsein C, Rotter S: Statistical properties of superimposed stationary spike trains. Journal of Computational Neuroscience 2012, 32(3):443-463.

5. Mazor O, Laurent G: Transient dynamics versus fixed points in odor representations by locust antennal lobe projection neurons. Neuron 2005, , 48: 661-673.

6. Silberberg G, Bethge M, Markram H, Pawelzik K, Tsodyks M: Dynamics of population rate codes in ensembles of neocortical neurons. Journal of Neurophysiology 2004, 91(2):704-709.

7. Deger M, Helias M, Cardanobile S, Atay FM, Rotter S: Nonequilibrium dynamics of stochastic point processes with refractoriness. Physical Review E 2010, 82:021129.

8. de la Rocha J, Doiron B, Shea-Brown E, Josic K, Reyes A: Correlation between neural spike trains increase with firing rate. Nature 2007, 448:802-806.

doi:10.1186/1471-2202-14-S1-P332

Cite this article as: Deniz and Rotter: Going beyond Poisson processes: a new statistical framework in neuronal modeling and data analysis. BMC Neuroscience 2013 14(Suppl 1):P332.

* Correspondence: taskin.deniz@bcf.uni-freiburg.de

Bernstein Center Freiburg \& Department of Biology, Freiburg University,

Freiburg, 79104, Germany 